\title{
WEIGHT CHARACTERIZATIONS FOR THE DISCRETE HARDY INEQUALITY WITH KERNEL
}

\author{
CHRISTOPHER A. OKPOTI, LARS-ERIK PERSSON, AND ANNA WEDESTIG
}

Received 16 August 2005; Accepted 17 August 2005

A discrete Hardy-type inequality $\left(\sum_{n=1}^{\infty}\left(\sum_{k=1}^{n} d_{n, k} a_{k}\right)^{q} u_{n}\right)^{1 / q} \leq C\left(\sum_{n=1}^{\infty} a_{n}^{p} v_{n}\right)^{1 / p}$ is considered for a positive "kernel" $d=\left\{d_{n, k}\right\}, n, k \in \mathbb{Z}_{+}$, and $p \leq q$. For kernels of product type some scales of weight characterizations of the inequality are proved with the corresponding estimates of the best constant $C$. A sufficient condition for the inequality to hold in the general case is proved and this condition is necessary in special cases. Moreover, some corresponding results for the case when $\left\{a_{n}\right\}_{n=1}^{\infty}$ are replaced by the nonincreasing sequences $\left\{a_{n}^{*}\right\}_{n=1}^{\infty}$ are proved and discussed in the light of some other recent results of this type.

Copyright (c) 2006 Christopher A. Okpoti et al. This is an open access article distributed under the Creative Commons Attribution License, which permits unrestricted use, distribution, and reproduction in any medium, provided the original work is properly cited.

\section{Introduction}

Let us consider the following special case of an interesting result in [4] (see also [5]) by Gol'dman.

Theorem 1.1. Let $0<r \leq p \leq q<\infty, \sigma=p r /(p-r)$ (for $p=r, \sigma=\infty)$. Then the inequality

$$
\left(\sum_{n=1}^{\infty}\left(\sum_{k=1}^{n}\left(a_{k} \varphi_{k}\right)^{r}\right)^{q / r} u_{n}^{q}\right)^{1 / q} \leq C\left(\sum_{n=1}^{\infty}\left(a_{n} v_{n}\right)^{p}\right)^{1 / p}
$$

for three weight sequences $\left\{\varphi_{n}\right\},\left\{u_{n}\right\}$, and $\left\{v_{n}\right\}(n=1,2, \ldots)$ holds if and only if the (Muckenhoupt type) condition

$$
B_{G}:=\sup _{n \geq 1}\left(\sum_{k=1}^{n}\left(\varphi_{k} v_{k}^{-1}\right)^{\sigma}\right)^{1 / \sigma}\left(\sum_{k=n}^{\infty} u_{k}^{q}\right)^{1 / q}<\infty .
$$

It holds with the usual maximum interpretation for the case $p=r(\sigma=\infty)$.

Hindawi Publishing Corporation Journal of Inequalities and Applications Volume 2006, Article ID 18030, Pages 1-14 DOI 10.1155/JIA/2006/18030 
Moreover, for the best constant $C$ in (1.1), $C \approx B_{G}$ (but without explicitly specifying the equivalence constants).

In this paper we will prove a result (see Corollary 3.3) showing that the Gol'dman condition (1.2) in fact can be replaced by some scales of conditions and also the estimate $C \approx B_{G}$ can be given in a much more precise form.

Partly guided by the development in the continuous case (see [6] and the literature therein) we will study the general inequality

$$
\left(\sum_{n=1}^{\infty}\left(\sum_{k=1}^{n} d_{n, k} a_{k}\right)^{q} u_{n}\right)^{1 / q} \leq C\left(\sum_{n=1}^{\infty} a_{n}^{p} v_{n}\right)^{1 / p}, \quad 1<p \leq q<\infty
$$

with a general kernel $d=\left\{d_{n, k}\right\}_{n, k=1}^{\infty}, d_{n, k} \geq 0$, involved.

We note that the first contribution in this direction was due to Andersen and Heinig [1, Theorem 4.1], who proved a sufficient condition for (1.3) to hold for the case $1 \leq p \leq$ $q<\infty$ with special nonnegative kernels $\left\{d_{n, k}\right\}_{n, k=1}^{\infty}$ that was assumed to be nonincreasing in $k$ and nondecreasing in $n$.

In this paper, using the result in [8] (see Proposition 2.2), we will prove some scales of characterizations for the special case with product weight kernel $d_{n, k}=l_{n} h_{k}, n, k=1,2, \ldots$ (see Theorem 3.1). Moreover, we will prove a sufficient condition also for the general case with an arbitrary nonnegative kernel (see Theorem 3.7), which at least for a special case is also necessary (see Remark 3.8).

Finally, partly guided by recent results by Sinnamon [12] (see also [11]), we will prove the surprising fact that we get the same characterizations in our Proposition 2.2 when restricting the set of positive sequences $\left\{a_{n}\right\}_{n=1}^{\infty}$ to the cone of nonincreasing sequences if, in addition, the weight sequence $\left\{v_{n}\right\}$ is nonincreasing (see Theorem 3.9).

The paper is organised as follows: in order not to disturb our discussions later on we present some preliminaries in Section 2. The main results together with some related remarks are presented in Section 3 and the proofs are given in Section 4. Finally, some concluding remarks and open questions can be found in Section 5.

\section{Preliminaries}

In this paper $\left\{a_{n}\right\}_{n=1}^{\infty}$ denotes an arbitrary (weight) sequence of nonnegative numbers. Moreover, $\left\{u_{n}\right\}_{n=1}^{\infty},\left\{v_{n}\right\}_{n=1}^{\infty},\left\{l_{n}\right\}_{n=1}^{\infty}$, and $\left\{h_{k}\right\}_{k=1}^{\infty}$ denote fixed weight sequences and $d=$ $\left\{d_{n, k}\right\}_{n, k=1}^{\infty}$ is a nonnegative discrete kernel, that is, a sequence of nonnegative numbers.

We will need the following technical lemma.

Lemma 2.1. Let $A_{k}=\sum_{n=1}^{k} a_{n}, A_{0}=0$ and, for $n=1,2, \ldots$, let $a_{n}>0$.

(a) If $0<\lambda<1$, then, for $k=1,2, \ldots$,

$$
\lambda A_{k}^{\lambda-1} a_{k} \leq A_{k}^{\lambda}-A_{k-1}^{\lambda} \leq \lambda A_{k-1}^{\lambda-1} a_{k} .
$$


(b) If $\lambda<0$ or $\lambda>1$, then, for $k=1,2, \ldots$,

$$
\lambda A_{k-1}^{\lambda-1} a_{k} \leq A_{k}^{\lambda}-A_{k-1}^{\lambda} \leq \lambda A_{k}^{\lambda-1} a_{k}
$$

Proof. The proof follows by using the mean value theorem in an appropriate way; for details see [8].

Recently Sinnamon [12] proved a remarkable result, which, in particular, means that some Hardy-type inequalities for nonincreasing sequences in fact are equivalent to the corresponding Hardy-type inequalities for general nonnegative sequences. Hence, they can be characterized by the same condition(s); see the books $[6,9]$ but also the more recent results, for example, in $[10,13,14]$. Here we also mention the following special case of a recent result in [8] (see also [7]), which we will need later on.

Proposition 2.2. Let $1<p \leq q<\infty$. Then the inequality

$$
\left(\sum_{n=1}^{\infty}\left(\sum_{k=1}^{n} a_{k}\right)^{q} u_{n}\right)^{1 / q} \leq C\left(\sum_{n=1}^{\infty} a_{n}^{p} v_{n}\right)^{1 / p}
$$

holds if and only if

$$
A_{1}(s):=\sup _{N \geq 1}\left(\sum_{n=1}^{N} v_{n}^{1-p^{\prime}}\right)^{s}\left(\sum_{n=N}^{\infty} u_{n}\left(\sum_{k=1}^{n} v_{k}^{1-p^{\prime}}\right)^{q\left(1 / p^{\prime}-s\right)}\right)^{1 / q}<\infty
$$

for some $s, 0<s \leq 1 / p^{\prime}$, or

$$
A_{2}(s):=\sup _{N \geq 1}\left(\sum_{n=1}^{N} v_{n}^{1-p^{\prime}}\right)^{-s}\left(\sum_{n=1}^{N} u_{k}\left(\sum_{k=1}^{n} v_{k}^{1-p^{\prime}}\right)^{q\left(1 / p^{\prime}+s\right)}\right)^{1 / q}<\infty,
$$

for some $s, 0<s \leq 1 / p$, or

$$
A_{3}(s):=\sup _{N \geq 1}\left(\sum_{n=N}^{\infty} u_{n}\right)^{s}\left(\sum_{n=1}^{N} v_{n}^{1-p^{\prime}}\left(\sum_{k=n}^{\infty} u_{k}\right)^{p^{\prime}(1 / q-s)}\right)^{1 / p^{\prime}}<\infty,
$$

for some $s, 0<s \leq 1 / q$, or

$$
A_{4}(s):=\sup _{N \geq 1}\left(\sum_{n=N}^{\infty} u_{n}\right)^{-s}\left(\sum_{n=N}^{\infty} v_{n}^{1-p^{\prime}}\left(\sum_{k=n}^{\infty} u_{k}\right)^{p^{\prime}(1 / q+s)}\right)^{1 / p^{\prime}}<\infty
$$

for some $s, 0<s \leq 1 / q^{\prime}$. 
4 Hardy's inequality

Moreover, for the best constant $C$ in (2.3), the following estimates hold:

$$
\begin{gathered}
\sup _{0<s<1 / p^{\prime}}\left(\frac{p s}{p s+1}\right)^{1 / p} A_{1}(s) \leq C \leq \inf _{0<s<1 / p^{\prime}} A_{1}(s)\left(\frac{p-1}{p(1-s)-1}\right)^{1 / p^{\prime}} \\
\sup _{0<s<1 / p}(p s)^{1 / p} A_{2}(s) \leq C \leq \frac{1}{(p-1)^{1 / q}}\left(\frac{q-p}{p \beta(p /(q-p), p(q-1) /(q-p))}\right)^{(q-p) / p q} A_{2}\left(\frac{1}{p}\right)
\end{gathered}
$$

if $p<q$ and

$$
A_{2}\left(\frac{1}{p}\right) \leq C \leq p^{\prime} A_{2}\left(\frac{1}{p}\right)
$$

if $p=q$,

$$
\begin{gathered}
\sup _{0<s<1 / q}\left(\frac{q^{\prime} s}{q^{\prime} s+1}\right)^{1 / q^{\prime}} A_{3}(s) \leq C \leq \inf _{0<s<1 / q} A_{3}(s)\left(\frac{q^{\prime}-1}{q^{\prime}(1-s)-1}\right)^{1 / q}, \\
\sup _{0<s<1 / q^{\prime}}\left(q^{\prime} s\right)^{1 / q^{\prime}} A_{4}(s) \\
\leq C \leq(q-1)^{1 / p^{\prime}}\left(\frac{q-p}{(p-1) q \beta(q /(q-p), q(p-1) /(q-p))}\right)^{(q-p) / p q} A_{4}\left(\frac{1}{q^{\prime}}\right)
\end{gathered}
$$

if $p<q$ and

$$
A_{4}\left(\frac{1}{q^{\prime}}\right) \leq C \leq p A_{4}\left(\frac{1}{q^{\prime}}\right)
$$

if $p=q$.

Remark 2.3. (a) The conditions $A_{3}(s)<\infty$ and $A_{4}(s)<\infty$ are just the natural duals of the conditions $A_{1}(s)<\infty$ and $A_{2}(s)<\infty$, respectively (cf. [6]).

(b) It is pointed out in [8] that as endpoint cases of some of the conditions above we just obtain some well-known conditions by Bennett (see $[2,3]$ ).

\section{Main results}

First we state the following generalization and unification of Theorem 1.1 and Proposition 2.2. 
Theorem 3.1. Let $1<p \leq q<\infty$ and consider the kernel $d=\left\{d_{n, k}\right\}_{n, k=1}^{\infty}$, where $d_{n, k}=l_{n} h_{k}$, $n, k=1,2, \ldots$. Then the inequality

$$
\left(\sum_{n=1}^{\infty}\left(\sum_{k=1}^{n} d_{n, k} a_{k}\right)^{q} u_{n}\right)^{1 / q} \leq C\left(\sum_{n=1}^{\infty} a_{n}^{p} v_{n}\right)^{1 / p}
$$

holds if and only if

$$
D_{1}(s):=\sup _{N \geq 1}\left(\sum_{n=1}^{N} h_{n}^{p^{\prime}} v_{n}^{1-p^{\prime}}\right)^{s}\left(\sum_{n=N}^{\infty} l_{n}^{q} u_{n}\left(\sum_{k=1}^{n} h_{k}^{p^{\prime}} v_{k}^{1-p^{\prime}}\right)^{q\left(1 / p^{\prime}-s\right)}\right)^{1 / q}<\infty,
$$

for some $s, 0<s \leq 1 / p^{\prime}$, or

$$
D_{2}(s):=\sup _{N \geq 1}\left(\sum_{n=1}^{N} h_{n}^{p^{\prime}} v_{n}^{1-p^{\prime}}\right)^{-s}\left(\sum_{n=1}^{N} l_{n}^{q} u_{n}\left(\sum_{k=1}^{n} h_{k}^{p^{\prime}} v_{k}^{1-p^{\prime}}\right)^{q\left(1 / p^{\prime}+s\right)}\right)^{1 / q}<\infty,
$$

for some $s, 0<s \leq 1 / p$, or

$$
D_{3}(s):=\sup _{N \geq 1}\left(\sum_{n=N}^{\infty} l_{n}^{q} u_{n}\right)^{s}\left(\sum_{n=1}^{N} h_{n}^{p^{\prime}} v_{n}^{1-p^{\prime}}\left(\sum_{k=n}^{\infty} l_{k}^{q} u_{k}\right)^{p^{\prime}(1 / q-s)}\right)^{1 / p^{\prime}}<\infty,
$$

for some $s, 0<s \leq 1 / q$, or

$$
D_{4}(s):=\sup _{N \geq 1}\left(\sum_{n=N}^{\infty} l_{n}^{q} u_{n}\right)^{-s}\left(\sum_{n=N}^{\infty} h_{n}^{p^{\prime}} v_{n}^{1-p^{\prime}}\left(\sum_{k=n}^{\infty} l_{k}^{q} u_{k}\right)^{p^{\prime}(1 / q+s)}\right)^{1 / p^{\prime}}<\infty,
$$

for some s, $0<s \leq 1 / q^{\prime}$.

Moreover, for the best constant $C$ in (3.1), the following estimates hold:

$$
\begin{gathered}
\sup _{0<s<1 / p^{\prime}}\left(\frac{p s}{p s+1}\right)^{1 / p} D_{1}(s) \leq C \leq \inf _{0<s<1 / p^{\prime}} D_{1}(s)\left(\frac{p-1}{p(1-s)-1}\right)^{1 / p^{\prime}} \\
\sup _{0<s<1 / p}(p s)^{1 / p} D_{2}(s) \leq C \leq \frac{1}{(p-1)^{1 / q}}\left(\frac{q-p}{p \beta(p /(q-p), p(q-1) /(q-p))}\right)^{(q-p) / p q} D_{2}\left(\frac{1}{p}\right)
\end{gathered}
$$

if $p<q$ and

$$
D_{2}\left(\frac{1}{p}\right) \leq C \leq p^{\prime} D_{2}\left(\frac{1}{p}\right)
$$


if $p=q$,

$$
\begin{gathered}
\sup _{0<s<1 / q}\left(\frac{q^{\prime} s}{q^{\prime} s+1}\right)^{1 / q^{\prime}} D_{3}(s) \leq C \leq \inf _{0<s<1 / q} D_{3}(s)\left(\frac{q^{\prime}-1}{q^{\prime}(1-s)-1}\right)^{1 / q}, \\
\sup _{0<s<1 / q^{\prime}}\left(q^{\prime} s\right)^{1 / q^{\prime}} D_{4}(s) \\
\leq C \leq(q-1)^{1 / p^{\prime}}\left(\frac{q-p}{(p-1) q \beta(q /(q-p), q(p-1) /(q-p))}\right)^{(q-p) / p q} D_{4}\left(\frac{1}{q^{\prime}}\right),
\end{gathered}
$$

if $p<q$ and

$$
D_{4}\left(\frac{1}{q^{\prime}}\right) \leq C \leq p D_{4}\left(\frac{1}{q^{\prime}}\right)
$$

if $p=q$.

Remark 3.2. For the case $d \equiv\{1\}$ we obtain Proposition 2.2 and we can also derive the following more precise version of Theorem 1.1.

Corollary 3.3. Let $0<r \leq p \leq q<\infty$ and $\sigma=p r /(p-r)$ (for $p=r, \sigma=\infty)$. Then the inequality (1.1) holds if and only if

$$
B_{1}(s)=\sup _{N \geq 1}\left(\sum_{n=1}^{N}\left(\varphi_{n} v_{n}^{-1}\right)^{\sigma}\right)^{s / r}\left(\sum_{n=N}^{\infty} u_{n}^{q}\left(\sum_{k=1}^{n}\left(\varphi_{k} v_{k}^{-1}\right)^{\sigma}\right)^{(q / r)(r / \sigma-s)}\right)^{1 / q}<\infty
$$

for some $s, 0<s \leq(r / \sigma)$.

Moreover, for the best constant $C$ in (1.1), the following estimates hold:

$$
\sup _{0<s<r / \sigma}\left(\frac{p s}{p s+r}\right)^{1 / p} B_{1}(s) \leq C \leq \inf _{0<s<r / \sigma}\left(\frac{p-r}{p(1-s)-r}\right)^{1 / \sigma} B_{1}(s) .
$$

Remark 3.4. If $s=r / \sigma$ in (3.12), then we have

$$
B_{1}\left(\frac{r}{\sigma}\right)=\sup _{n \geq 1}\left(\sum_{k=1}^{n}\left(\varphi_{k} v_{k}^{-1}\right)^{\sigma}\right)^{1 / \sigma}\left(\sum_{k=n}^{\infty} u_{k}^{q}\right)^{1 / q}<\infty,
$$

which coincides with (1.2) (i.e., $\left.B_{1}(r / \sigma)=B_{G}\right)$ and the statement in Theorem 1.1 follows. Remark 3.5. Remark 3.4 means that the scale of conditions in Corollary 3.3 has the Gol'dman condition in its right endpoint. However, there exist also other scales of conditions of completely different types for characterizing (1.1). See [7], our Remark 5.5, and Example 5.6. 
Remark 3.6. When $r=1$ and $\varphi_{k}=1, k=1,2, \ldots$, in Corollary 3.3, then the inequality (1.1) with $v_{n}$ replaced by $v_{n}^{1 / p}$ and $u_{n}$ replaced by $u_{n}^{1 / q}$ coincides with (2.3). In particular, for the case $s=1 / p^{\prime}$ in (3.12), we have

$$
B_{1}\left(\frac{1}{p^{\prime}}\right)=\sup _{n \geq 1}\left(\sum_{k=1}^{n} v_{k}^{1-p^{\prime}}\right)^{1 / p^{\prime}}\left(\sum_{k=n}^{\infty} u_{k}\right)^{1 / q}<\infty,
$$

which coincides with Muckenhoupt's condition $A_{1}\left(1 / p^{\prime}\right)<\infty$ (cf. (2.4) and also Bennett [2]).

Next we state the following result for the case with a general kernel.

Theorem 3.7. Let $1<p \leq q<\infty$. If

$$
E(s):=\sup _{N \geq 1}\left(\sum_{n=1}^{N} v_{n}^{1-p^{\prime}}\right)^{s}\left(\sum_{n=N}^{\infty} d_{n, k}^{q} u_{n}\left(\sum_{m=1}^{n} v_{m}^{1-p^{\prime}}\right)^{q\left(1 / p^{\prime}-s\right)}\right)^{1 / q}<\infty
$$

holds for some $s \in\left(0,1 / p^{\prime}\right)$, then the inequality (1.3) holds with

$$
C \leq \inf _{0<s<1 / p^{\prime}}\left(\frac{p-1}{p-s p-1}\right)^{1 / p^{\prime}} E(s) .
$$

Remark 3.8. For the case $d_{n, k}=1, n, k=1,2, \ldots$, the condition (3.16) coincides with the condition (2.4) and, thus, according to Proposition 2.2, in this case the condition (3.16) is both necessary and sufficient for the inequality (1.3) to hold.

Inspired by a recent result of Sinnamon [12], we also state the following.

Theorem 3.9. Let $1<p \leq q<\infty$. Then the inequality

$$
\left(\sum_{n=1}^{\infty}\left(\sum_{k=1}^{n} a_{k}^{*}\right)^{q} u_{n}\right)^{1 / q} \leq C\left(\sum_{n=1}^{\infty}\left(a_{n}^{*}\right)^{p} v_{n}\right)^{1 / p}
$$

holds for all nonincreasing sequences $\left\{a_{n}^{*}\right\}_{n=1}^{\infty}$ with the additional condition that $\left\{v_{n}\right\}_{n=1}^{\infty}$ is nonincreasing if and only if the condition (2.4) holds. Moreover, for the best constant $C$ in (3.18), the estimate (2.8) holds.

Remark 3.10. For the case $v_{n}=1, n=1,2, \ldots$, the statement in Theorem 3.9 is a special case of a recent remarkable result of Sinnamon [12, pages 300-301].

\section{Proofs}

Proof of Theorem 3.1. With the kernel $\left\{d_{n, k}\right\}=\left\{l_{n} h_{k}\right\}$ the inequality (3.1) becomes

$$
\left(\sum_{n=1}^{\infty}\left(\sum_{k=1}^{n} l_{n} h_{k} a_{k}\right)^{q} u_{n}\right)^{1 / q} \leq C\left(\sum_{n=1}^{\infty} a_{n}^{p} v_{n}\right)^{1 / p}
$$


that is,

$$
\left(\sum_{n=1}^{\infty}\left(\sum_{k=1}^{n} h_{k} a_{k}\right)^{q} l_{n}^{q} u_{n}\right)^{1 / q} \leq C\left(\sum_{n=1}^{\infty} a_{n}^{p} v_{n}\right)^{1 / p} .
$$

We now put $b_{k}=h_{k} a_{k}$ in the inequality (4.2) and note that (4.2) is equivalent to

$$
\left(\sum_{n=1}^{\infty}\left(\sum_{k=1}^{n} b_{k}\right)^{q} l_{n}^{q} u_{n}\right)^{1 / q} \leq C\left(\sum_{n=1}^{\infty} b_{n}^{p} h_{n}^{-p} v_{n}\right)^{1 / p}
$$

Considering $l_{n}^{q} u_{n}=\tilde{u}_{n}$ and $h_{n}^{-p} v_{n}=\tilde{v}_{n}$ to be our new fixed nonnegative weight sequences, we have that the inequality

$$
\left(\sum_{n=1}^{\infty}\left(\sum_{k=1}^{n} b_{k}\right)^{q} \tilde{u}_{n}\right)^{1 / q} \leq C\left(\sum_{n=1}^{\infty} b_{n}^{p} \tilde{v}_{n}\right)^{1 / p}
$$

is equivalent to the Hardy-type inequality (2.3). Thus, by replacing $u_{n}$ by $l_{n}^{q} u_{n}$ and $v_{n}$ by $h_{n}^{-p} v_{n}$ in the conditions (2.4)-(2.7) (i.e., those described by $\left.A_{1}(s)-A_{4}(s)\right)$ and using Proposition 2.2, we obtain that the conditions (3.2)-(3.5) (i.e., those described by $D_{1}(s)-$ $D_{4}(s)$ ) are necessary and sufficient conditions for (4.4), and, thus, (3.1) to hold. Subsequently, by replacing $A_{i}(s)$ with $D_{i}(s), i=1, \ldots, 4$, respectively, in the estimates $(2.8)$ (2.2), we obtain the estimates for the best constant $C$ in (3.1) to be those described in (3.6)-(3.10). The proof is complete.

Proof of Corollary 3.3. In the inequality (3.1) with $d_{n, k}=l_{n} h_{k}$, we let $h_{k}=\varphi_{k}^{r}$ and let $l_{n}=$ $u_{n}^{(q r-1) / q}$ and replace $a_{n}$ with $a_{n}^{r}$ and $v_{n}$ with $v_{n}^{p r}$ :

$$
\left(\sum_{n=1}^{\infty}\left(\sum_{k=1}^{n} \varphi_{k}^{r} a_{k}^{r}\right)^{q} u_{n}^{q r}\right)^{1 / q} \leq C\left(\sum_{n=1}^{\infty} a_{n}^{p r} v_{n}^{p r}\right)^{1 / p}
$$

Moreover, replace $p$ with $p / r$ and $q$ with $q / r$, and we obtain

$$
\left(\sum_{n=1}^{\infty}\left(\sum_{k=1}^{n} \varphi_{k}^{r} a_{k}^{r}\right)^{q / r} u_{n}^{q}\right)^{1 / q} \leq C_{o}\left(\sum_{n=1}^{\infty} a_{n}^{p} v_{n}^{p}\right)^{1 / p}
$$

with $C_{o}=C^{1 / r}$ which is equivalent to the inequality (1.1).

This means that for the case $0<r<p \leq q<\infty$, we can characterize the inequality (1.1) by using Theorem 3.1. Thus, in condition (3.2) we first let $l_{n}=u_{n}^{(q r-1) / q}, h_{n}=\varphi_{n}^{r}, v_{n}=v_{n}^{p r}$, after that replace $p$ by $p / r$ and $q$ by $q / r$, and finally raise the condition to the power $1 / r$. 
Hence, by Theorem 3.1, we conclude that the condition (3.12) (i.e., that described by $B_{1}(s)$ ) characterizes (1.1). Moreover, the estimate (3.13) follows in a similar way from the estimate (3.6). The proof is complete.

Proof of Theorem 3.7. Put $b_{n}^{p}=a_{n}^{p} v_{n}$ in (1.3). Then (1.3) is equivalent to

$$
\left(\sum_{n=1}^{\infty}\left(\sum_{k=1}^{n} d_{n, k} b_{k} v_{k}^{-1 / p}\right)^{q} u_{n}\right)^{1 / q} \leq C\left(\sum_{n=1}^{\infty} b_{n}^{p}\right)^{1 / p} .
$$

Assume that the condition (3.16) holds and let

$$
V_{n}=\sum_{k=1}^{n} v_{k}^{1-p^{\prime}}
$$

Applying Hölder's inequality, Lemma 2.1(a) with $a_{k}=v_{k}^{1-p^{\prime}}(0<\lambda=(p-s p-1) /(p-$ $1)<1$ ), and Minkowski's inequality to the left-hand side of $(4.7)$, we find that

$$
\begin{aligned}
\left(\sum_{n=1}^{\infty}\right. & \left.\left(\sum_{k=1}^{n} d_{n, k} b_{k} v_{k}^{-1 / p}\right)^{q} u_{n}\right)^{1 / q} \\
& =\left(\sum_{n=1}^{\infty}\left(\sum_{k=1}^{n} d_{n, k} b_{k} V_{k}^{s} V_{k}^{-s} v_{k}^{-1 / p}\right)^{q} u_{n}\right)^{1 / q} \\
& \leq\left(\sum_{n=1}^{\infty}\left(\sum_{k=1}^{n} d_{n, k}^{p} b_{k}^{p} V_{k}^{s p}\right)^{q / p}\left(\sum_{k=1}^{n} V_{k}^{-s p^{\prime}} v_{k}^{-p^{\prime} / p}\right)^{q / p^{\prime}} u_{n}\right)^{1 / q} \\
& =\left(\sum_{n=1}^{\infty}\left(\sum_{k=1}^{n} d_{n, k}^{p} b_{k}^{p} V_{k}^{s p}\right)^{q / p}\left(\sum_{k=1}^{n} V_{k}^{-s p /(p-1)} v_{k}^{1-p^{\prime}}\right)^{q / p^{\prime}} u_{n}\right)^{1 / q} \\
& \leq\left(\frac{p-1}{p-s p-1}\right)^{1 / p^{\prime}}\left(\sum_{n=1}^{\infty}\left(\sum_{k=1}^{n} d_{n, k}^{p} b_{k}^{p} V_{k}^{s p}\right)^{q / p} V_{n}^{q((p-s p-1) / p)} u_{n}\right)^{1 / q} \\
& \leq\left(\frac{p-1}{p-s p-1}\right)^{1 / p^{\prime}}\left(\sum_{k=1}^{\infty} b_{k}^{p} V_{k}^{s p}\left(\sum_{n=k}^{\infty} d_{n, k}^{q} V_{n}^{q\left(1 / p^{\prime}-s\right)} u_{n}\right)^{p / q}\right)^{1 / p} \\
& \leq\left(\frac{p-1}{p-s p-1}\right)^{1 / p^{\prime}} \sup _{k \geq 1} V_{k}^{s}\left(\sum_{n=k}^{\infty} d_{n, k}^{q} V_{n}^{q\left(1 / p^{\prime}-s\right)} u_{n}\right)^{1 / q}\left(\sum_{k=1}^{\infty} b_{k}^{p}\right)^{1 / p} .
\end{aligned}
$$

Hence, (4.7), and, thus, (1.3) hold. By taking infimum over $s \in\left(0,1 / p^{\prime}\right)$, we find that also (3.17) holds. The proof is complete.

Proof of Theorem 3.9

Sufficiency. The proof follows by just using Proposition 2.2 in the present situation and also the upper estimate in (2.8) is obtained. However, here we make the following independent proof. 
Assume that the condition (2.4) holds and let $\left\{a_{n}^{*}\right\}_{n=1}^{\infty}$ be an arbitrary nonincreasing sequence and define $a_{n}^{*}=\left(\sum_{m=n}^{\infty} t_{m}\right)^{1 / p}, n=1,2, \ldots$. The inequality (3.18) can equivalently be rewritten as

$$
\left(\sum_{n=1}^{\infty}\left(\sum_{k=1}^{n}\left(\sum_{m=k}^{\infty} t_{m}\right)^{1 / p}\right)^{q} u_{n}\right)^{1 / q} \leq C\left(\sum_{n=1}^{\infty}\left(\sum_{m=n}^{\infty} t_{m}\right) v_{n}\right)^{1 / p}=C\left(\sum_{m=1}^{\infty} t_{m} \sum_{n=1}^{m} v_{n}\right)^{1 / p}
$$

Taking $V_{n}$ as it is defined in (4.8) and applying Hölder's inequality, Lemma 2.1(a) (with $\lambda=(p-s p-1) /(p-1))$, Minkowski's inequality, and changing the order of the summation to the left-hand side of (4.10), we have that

$$
\begin{aligned}
\left(\sum_{n=1}^{\infty}\right. & \left.\left(\sum_{k=1}^{n}\left(\sum_{m=k}^{\infty} t_{m}\right)^{1 / p}\right)^{q} u_{n}\right)^{1 / q} \\
& =\left(\sum_{n=1}^{\infty}\left(\sum_{k=1}^{n}\left(\sum_{m=k}^{\infty} t_{m}\right)^{1 / p} V_{k}^{s} V_{k}^{-s} v_{k}^{1 / p} v_{k}^{-1 / p}\right)^{q} u_{n}\right)^{1 / q} \\
& \leq\left(\sum_{n=1}^{\infty}\left(\sum_{k=1}^{n}\left(\sum_{m=k}^{\infty} t_{m}\right) V_{k}^{s p} v_{k}\right)^{q / p}\left(\sum_{k=1}^{n} V_{k}^{-s p /(p-1)} v_{k}^{1-p^{\prime}}\right)^{q / p^{\prime}} u_{n}\right)^{1 / q} \\
& \leq\left(\frac{p-1}{p-s p-1}\right)^{1 / p^{\prime}}\left(\sum_{n=1}^{\infty}\left(\sum_{k=1}^{n}\left(\sum_{m=k}^{\infty} t_{m}\right)_{k}^{s p} v_{k}\right)^{q / p} V_{n}^{q(p-s p-1) / p} u_{n}\right)^{1 / q} \\
& \leq\left(\frac{p-1}{p-s p-1}\right)^{1 / p^{\prime}}\left(\sum_{k=1}^{\infty}\left(\sum_{m=k}^{\infty} t_{m}\right)_{k}^{s p} v_{k}\left(\sum_{n=k}^{\infty} V_{n}^{q(p-s p-1) / p} u_{n}\right)^{p / q}\right)^{1 / p} \\
& =\left(\frac{p-1}{p-s p-1}\right)^{1 / p^{\prime}}\left(\sum_{m=1}^{\infty} t_{m} \sum_{k=1}^{m} v_{k} V_{k}^{s p}\left(\sum_{n=k}^{\infty} V_{n}^{q\left(1 / p^{\prime}-s\right)} u_{n}\right)^{p / q}\right)^{1 / p} \\
& \leq\left(\frac{p-1}{p-s p-1}\right)^{1 / p^{\prime}} \sup _{k \geq 1} V_{k}^{s}\left(\sum_{n=k}^{\infty} V_{n}^{q\left(1 / p^{\prime}-s\right)} u_{n}\right)^{1 / q}\left(\sum_{m=1}^{\infty} t_{m} \sum_{k=1}^{m} v_{k}\right)^{1 / p} .
\end{aligned}
$$

Hence, by taking infimum over $s \in\left(0,1 / p^{\prime}\right),(4.10)$, and thus, (3.18) hold with a constant $C$ satisfying the right-hand inequality in (2.8).

Necessity. Assume that (3.18) holds and for fixed $N \in \mathbb{Z}_{+}$apply the following test sequence:

$$
a_{k}^{*}= \begin{cases}V_{N}^{-((1+p s) / p)} v_{k}^{1-p^{\prime}} & \text { for } k=1, \ldots, N \\ V_{k}^{-((1+p s) / p)} v_{k}^{1-p^{\prime}} & \text { for } k=N+1, \ldots\end{cases}
$$

to (3.18). (Note that with our assumptions $\left\{a_{k}^{*}\right\}_{k=1}^{\infty}$ is a nonincreasing sequence.) 
For the left-hand side of (3.18) we have that

$$
\begin{aligned}
\left(\sum_{n=1}^{\infty}\right. & \left.\left(\sum_{k=1}^{n} a_{k}^{*}\right)^{q} u_{n}\right)^{1 / q} \\
& =\left(\sum_{n=1}^{\infty}\left(\sum_{k=1}^{N} V_{N}^{-((1+p s) / p)} v_{k}^{1-p^{\prime}}+\sum_{k=N+1}^{n} V_{k}^{-((1+p s) / p)} v_{k}^{1-p^{\prime}}\right)^{q} u_{n}\right)^{1 / q} \\
& \geq\left(\sum_{n=N}^{\infty}\left(\sum_{k=1}^{N} V_{N}^{-((1+p s) / p)} v_{k}^{1-p^{\prime}}+\sum_{k=N+1}^{n} V_{k}^{-((1+p s) / p)} v_{k}^{1-p^{\prime}}\right)^{q} u_{n}\right)^{1 / q} \\
& \left.\geq\left(\sum_{n=N}^{\infty}\left(V_{N}^{1 / p^{\prime}-s}+V_{n}^{-(1+p s) / p} \sum_{k=N+1}^{n} v_{k}^{1-p^{\prime}}\right)^{q} u_{n}\right)^{1 / q}\right)^{1 / q} \\
& =\left(\sum_{n=N}^{\infty}\left(V_{N}^{1 / p^{\prime}-s}+V_{n}^{-(1+p s) / p}\left(V_{n}-V_{N}\right)\right)^{q} u_{n}\right)^{1 / q} \\
& \geq\left(\sum_{n=N}^{\infty}\left(V_{N}^{1 / p^{\prime}-s}+V_{n}^{1 / p^{\prime}-s}-V_{N}^{-(1+p s) / p} V_{N}\right)^{q} u_{n}\right)^{1 / q} \\
& =\left(\sum_{n=N}^{\infty} V_{n}^{q\left(1 / p^{\prime}-s\right)} u_{n}\right)^{1 /}
\end{aligned}
$$

For the right-hand side of (3.18), by applying Lemma 2.1(b) we find that

$$
\begin{aligned}
\left(\sum_{n=1}^{\infty}\left(a_{n}^{*}\right)^{p} v_{n}\right)^{1 / p} & =\left(\sum_{n=1}^{N} V_{N}^{-(1+p s)} v_{k}^{1-p^{\prime}}+\sum_{n=N+1}^{\infty} V_{k}^{-(1+p s)} v_{k}^{1-p^{\prime}}\right)^{1 / p} \\
& \leq\left(V_{N}^{-p s}+\frac{1}{p s} V_{N}^{-p s}\right)^{1 / p} \\
& \leq\left(1+\frac{1}{p s}\right)^{1 / p} V_{N}^{-s}=\left(\frac{p s+1}{p s}\right)^{1 / p} V_{N}^{-s} .
\end{aligned}
$$

Combining (4.14), (4.13), and (3.18) we have that

$$
\left(\sum_{n=N}^{\infty} V_{n}^{q\left(1 / p^{\prime}-s\right)} u_{n}\right)^{1 / q} \leq C\left(\frac{p s+1}{p s}\right)^{1 / p} V_{N}^{-s}
$$

that is, that

$$
\left(\frac{p s}{p s+1}\right)^{1 / p} V_{N}^{s}\left(\sum_{n=N}^{\infty} V_{n}^{q\left(1 / p^{\prime}-s\right)} u_{n}\right)^{1 / q} \leq C<\infty .
$$


Hence, by taking supremum over $N \geq 1$ and supremum over $s \in\left(0,1 / p^{\prime}\right)$, we conclude that (2.4) and the left-hand side of the estimate (2.8) hold.

Summing up, we have proved that (3.18) is equivalent to (2.4) and that (2.8) holds. The proof is complete.

\section{Concluding remarks}

By comparing the statements in Theorem 3.7 and Remark 3.8 and the corresponding knowledge from the continuous case (see [6]), it is natural to raise the following question.

Open question 5.1. Find necessary and sufficient conditions for (1.3) to hold for all nonnegative sequences $\left\{a_{n}\right\}_{n=1}^{\infty}$ for as general kernels as possible.

Remark 5.2. For the case $v_{n}=1, n=1,2, \ldots$ Proposition 2.2 holds also if the set of all weight sequences $\left\{a_{n}\right\}_{n=1}^{\infty}$ is restricted to the cone of all nonincreasing weight sequences $\left\{a_{n}^{*}\right\}_{n=1}^{\infty}$ (cf. also Remark 3.10). This fact follows from a recent result of Sinnamon [12].

Remark 5.3. The result of Sinnamon [12] was recently generalized by Persson et al. [11] to a more general case involving kernels and general measures. However, these kernels still have some restrictions (monotonicity in the second variable). These results make it natural to also raise the following question.

Open question 5.4. Find necessary and sufficient conditions for (1.3) to hold on the cone of nonincreasing sequences for as general kernels as possible.

Remark 5.5. The statement in Corollary 3.3 follows by using just the conditions (3.2) and (3.6) in Theorem 3.1. By using the other (equivalent) conditions, we can obtain other scales of conditions for characterizing (1.1). See [7]; here we just give the following example.

Example 5.6. Let $0<r \leq p \leq q<\infty$ and $\sigma=p r /(p-r)$ (for $p=r, \sigma=\infty$ ). Then the inequality (1.1) holds if and only if

$$
B_{2}(s)=\sup _{N \geq 1}\left(\sum_{n=1}^{N}\left(\varphi_{n} v_{n}^{-1}\right)^{\sigma}\right)^{-s / r}\left(\sum_{n=N}^{\infty} u_{n}^{q}\left(\sum_{k=1}^{n}\left(\varphi_{k} v_{k}^{-1}\right)^{\sigma}\right)^{(q / r)(r / \sigma+s)}\right)^{1 / q}<\infty
$$

for some $s, 0<s \leq r / p$.

Moreover, for the best constant $C$ in (1.1) we have the following estimates:

$$
\sup _{0<s<r / p}\left(\frac{p}{r} s\right)^{1 / p} B_{2}(s) \leq C \leq\left(\frac{\sigma}{p}\right)^{1 / q}\left(\frac{q-p}{p \beta(p /(q-p), p(q-r) / r(q-p))}\right)^{(q-p) / p q} B_{2}\left(\frac{r}{p}\right)
$$


if $p<q$ and

$$
B_{2}\left(\frac{r}{p}\right) \leq C \leq \frac{p}{p-r} B_{2}\left(\frac{r}{p}\right)
$$

if $p=q$.

Remark 5.7. In $[4,5]$ by Gol'dman the case $p>q$ was also treated and the results in this paper indicate that the results of Gol'dman can be generalized in a similar way also for this case. We aim to return to this question in a forthcoming paper.

\section{Acknowledgment}

We thank Professor Saburoh Saitoh for some generous suggestions which have improved the final version of this paper.

\section{References}

[1] K. F. Andersen and H. P. Heinig, Weighted norm inequalities for certain integral operators, SIAM Journal on Mathematical Analysis 14 (1983), no. 4, 834-844.

[2] G. Bennett, Some elementary inequalities, The Quarterly Journal of Mathematics. Oxford. Second Series 38 (1987), no. 152, 401-425.

[3] _ Some elementary inequalities. III, The Quarterly Journal of Mathematics. Oxford. Second Series 42 (1991), no. 166, 149-174.

[4] M. L. Gol'dman, Hardy type inequalities on the cone of quasi-monotone functions, Research report 98/31, Russian Academy of Sciences Far-Eastern Branch Computer Center, Khabarovsk, 1998, 70 pages.

[5] Estimates for the norms of integral and discrete operators of Hardy type on cones of quasimonotone functions, Doklady Akademii Nauk 377 (2001), no. 6, 733-738 (Russian).

[6] A. Kufner and L.-E. Persson, Weighted Inequalities of Hardy Type, World Scientific, New Jersey, 2003.

[7] C. A. Okpoti, Weight characterization of discrete Hardy and Carleman type inequalities, Licentiate thesis, Department of Mathematics, Luleå University of Technology, Luleå, 2005, in print.

[8] C. A. Okpoti, L.-E. Persson, and A. Wedestig, Scales of weight characterizations for the discrete Hardy and Carleman inequalities, Proceedings of Function Spaces, Differential Operators and Nonlinear Analysis (FSDONA '04), Academy of Sciences of the Czech Republic, Milovy, 2004, pp. 236-258.

[9] B. Opic and A. Kufner, Hardy-Type Inequalities, Pitman Research Notes in Mathematics Series, vol. 219, Longman Scientific \& Technical, Harlow, 1990.

[10] L.-E. Persson and V. D. Stepanov, Weighted integral inequalities with the geometric mean operator, Journal of Inequalities and Applications 7 (2002), no. 5, 727-746, an abbreviated version can also be found in Russian Academy of Sciences. Doklady. Mathematics 63 (2001), 201-202.

[11] L.-E. Persson, V. D. Stepanov, and E. P. Ushakova, Equivalence of Hardy-type inequalities with general measures on the cones of non-negative respective non-increasing functions, to appear in Proceedings of the American Mathematical Society.

[12] G. Sinnamon, Hardy's inequality and monotonicity, Proceedings of Function Spaces, Differential Operators and Nonlinear Analysis (FSDONA '04), Academy of Sciences of the Czech Republic, Milovy, 2004, pp. 292-310.

[13] A. Wedestig, Some new Hardy type inequalities and their limiting inequalities, JIPAM. Journal of Inequalities in Pure and Applied Mathematics 4 (2003), no. 3, 15, article 61. 


\section{Hardy's inequality}

[14] Weighted inequalities of Hardy-type and their limiting inequalities, Ph.D. thesis, Department of Mathematics, Luleå University of Technology, Luleå, 2003, 106 pages.

Christopher A. Okpoti: Department of Mathematics, Luleå University of Technology,

97187 Luleå, Sweden

E-mail address: cao@sm.luth.se

Lars-Erik Persson: Department of Mathematics, Luleå University of Technology,

97187 Luleå, Sweden

E-mail address: larserik@sm.luth.se

Anna Wedestig: Department of Mathematics, Luleå University of Technology,

97187 Luleå, Sweden

E-mail address: annaw@sm.luth.se 